\title{
X-RAY DIFFRACTION METHOD FOR DETERMINATION OF TEXTURE EVOLUTION IN LAYERS
}

\author{
I. TOMOV, R. BANOVA, S. SURNEV \\ Institute of Physical Chemistry, Bulgarian Academy of Sciences, 1040 Sofia, \\ Bulgaria
}

(Received October 1, 1991)

\begin{abstract}
An X-ray diffraction method for the determination of texture evolution in electrodeposited and vapour-deposited layers was developed. It was applied to the study of the texture of electrodeposited bright zinc. It was established that the arising of texture in the concrete models is due to oriented nucleation as well as to a growth selection process. The substrate structure also has an effect on the character of the texture evolution.
\end{abstract}

KEY WORDS texture evolution, X-ray diffraction method, growth selection process, layers, electrodeposition, zinc.

\section{INTRODUCTION}

It is well known that the texture arising in layers is related to continuous microstructural changes in normal direction to the substrate. On the basis of proposed classification (Bauer 1956, Reimer and Freking 1965) van der Drift, 1967, distinguished two contradictory ways of how the texture sharpness changes with the layer thickness. In the case of oriented nucleation, no matter whether the initial preferred orientation has arisen on a single crystal or a polycrystalline substrate, the texture sharpness decreases during the vertical growth. In the case of oriented growth, the texture sharpness increases during vertical growth. The latter is a result of the growth selection process, due to the growth rate differences of the variously oriented crystallites.

The mechanism of texture formation could be revealed if experiments were carried out in which the microstructural changes in some crystal directions were monitored during the layer growth (or even just in some stages of its growth). Thus the aim of the present study is to specify the X-ray diffraction method suitable for controlling the structural changes with the rise in layer thickness. Its application for the study of the texture evolution of electrodeposited zinc would yield information on the mechanism of its arising in the particular case. The comparing of the newly obtained characteristics with known standpoints and results concerning the arising of the texture undoubtedly will widen our perspectives of this problem. 


\section{EXPRESSING THE POLE DENSITY OF A THIN SURFACE SUBLAYER}

The texture evolution can be traced by means of $\mathrm{X}$-ray diffraction control of pole density in ideal directions of main texture components. We assume that we know the increase in the thickness $\Delta t_{1}, \Delta t_{2}, \Delta t_{3}, \ldots, \Delta t_{m}, \ldots, \Delta t_{n}$ of the textured layer in each stage of its growth. Each $\Delta t_{m}$ defines the thickness of the respective $m$-sublayer. The sublayer thickness has to be less than the operative information depth of X-ray penetration (Tomov and Bunge 1991). To solve the problem it is necessary to know the individual diffraction contribution of each sublayer to the measured integrated intensity. It is possible by means of $\mathrm{X}$-ray diffraction to determine only the mean value of the pole density of a given sublayer. Thus the proposed model contains the approximation that the pole density inside the thin sublayer does not change with its thickness, i.e. the structural changes come about with a jump at the border between two adjacent sublayers.

Let us denote by $I_{1}$ the integrated intensity diffracted from the first sublayer $\Delta t_{1}$, located on the substrate (Figure 1a). If a layer with $\Delta t_{2}$ is grown over it (Figure $2 b$ ), then the total intensity $I_{1,2}$, diffracted from the two sublayers will be expressed by

$$
I_{1,2}=I_{2}+I_{1} \exp \left(-\frac{2 \mu}{\sin \theta} \Delta t_{2}\right)
$$

where $I_{2}$ is the integrated intensity diffracted by the second sublayer with a thickness of $\Delta t_{2}$, while

$$
I_{1} \exp \left(-\frac{2 \mu}{\sin \theta} \Delta t_{2}\right)
$$

is the contribution to the intensity $I_{1,2}$, due to the first sublayer which is covered by the sublayer $\Delta t_{2}$. The factor

$$
\exp \left(-\frac{2 \mu}{\sin \theta} \Delta t_{2}\right)
$$
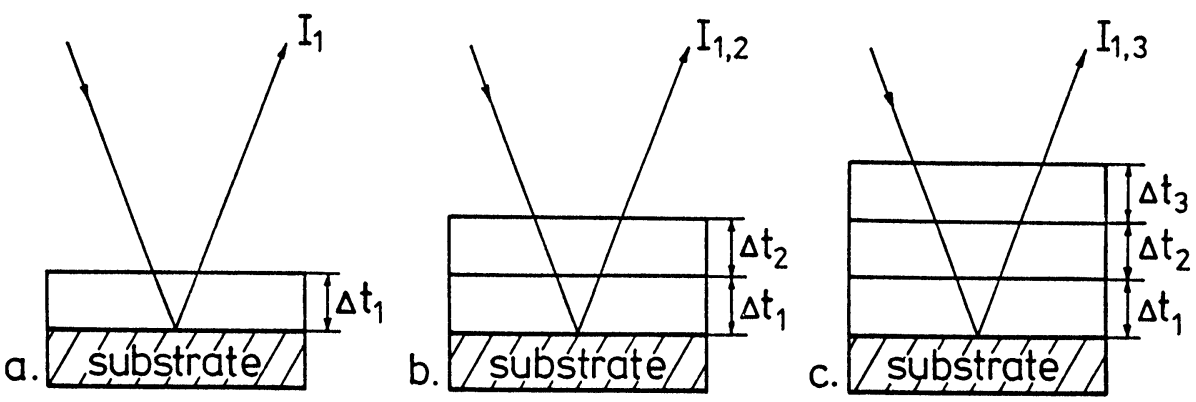

Figure 1 A schematic presentation of the diffraction by layers, the total thickness is less than the operative information depth of X-ray penetration. The integrated intensities $I$, diffracted by: a) one sublayer $\left(I_{1}\right)$; b) two sublayers $\left(I_{1,2}\right)$; c) three sublayers $\left(I_{1,3}\right)$ with thicknesses of $\Delta t_{1}, \Delta t_{2}$ and $\Delta t_{3}$, respectively. 
accounts for the attenuating of the $\mathrm{X}$-ray intensity as a result of their absorption in a layer with a thickness of $\Delta t_{2}$.

If a third sublayer is grown with $\Delta t_{3}$ (Figure 1c), the total intensity measured $I_{1,3}$ is expressed by

$$
I_{1,3}=I_{3}+I_{2} \exp \left(-\frac{2 \mu}{\sin \theta} \Delta t_{3}\right)+I_{1} \exp \left[-\frac{2 \mu}{\sin \theta}\left(\Delta t_{2}+\Delta t_{3}\right)\right]
$$

In the general case of a layer which has been obtained by the growth of $n$ sublayers, the total intensity $I_{1, n}$ is a superposition of the diffraction contributions of all sublayers, i.e.

or

$$
\begin{aligned}
I_{1, n}= & I_{n-1} \exp \left[-\frac{2 \mu}{\sin \theta}\left(\Delta t_{n}\right)\right] \\
& +I_{n-2} \exp \left[-\frac{2 \mu}{\sin \theta}\left(\Delta t_{n}+\Delta t_{n-1}\right)\right] \\
& \left.+\frac{2 \mu}{\sin \theta}\left(\sum_{k=m+1}^{n} \Delta t_{k}\right)\right] \\
& +I_{m} \exp \left[-\frac{2 \mu}{\sin \theta}\left(\sum_{k=2}^{n} \Delta t_{k}\right)\right] \\
& +I_{1} \exp [-
\end{aligned}
$$

$$
I_{1, n}=I_{n}+\sum_{m=1}^{n-1} I_{m} \exp \left[-\frac{2 \mu}{\sin \theta}\left(\sum_{k=m+1}^{n} \Delta t_{k}\right)\right]
$$

where the right-hand side of Eq. (4) shows the contributions of all $m$-sublayers in the interval $[1 \leq m \leq=n]$ to the intensity $I_{1, n}$. Experimentally measurable are $I_{1}$ and all intensities $I_{1, n}$.

The intensity $I_{n}$, diffracted only by the surface sublayer is expressed by

$$
I_{n}=I_{1, n}-\sum_{m=1}^{n-1} I_{m} \exp \left[-\frac{2 \mu}{\sin \theta}\left(\sum_{k=m+1}^{n} \Delta t_{k}\right)\right]
$$

$I_{n}$ depends both on the surface sublayer thickness and the pole density in the respective direction. Then the integrated intensity $I_{n}^{\infty}$ of an "infinitely" thick layer, having the same structure as the $n$-th sublayer is expressed by

$$
I_{n}^{\infty}=\frac{I_{n}}{1-\exp \left(-\frac{2 \mu \Delta t_{n}}{\sin \theta}\right)}
$$

Its value does not depend on the sublayer thickness. The knowledge of $I_{n}^{\infty}$ allows us to express the pole density $P_{n}$ in the respective crystal direction

$$
I_{n}^{\infty}=P_{n} N
$$

where $N$ is the normalization factor of the pole figure. Its value, in the case of a diffractometer, may be determined by employing the approach described by Tomov, 1986, which requires a joint determination of $P_{n}$ and $N$ from an incompletely or completely measured pole figure (Tomov and Bunge 1979) of an 
"infinitely" thick layer. In such a way the pole density $P_{n}$ of each successively grown $n$-th thin surface sublayer was determined.

An averaged value will be obtained as a result of the experimental measurements of the pole density of each sublayer. It is evident that the thinner the sublayers are, the less "smeared out" the experimental results for the respective pole densities will be. An absolutely correct information for the texture evolution would be obtained in case of "infinitely" thin sublayers. In practice this could be carried out if the X-ray diffraction measurement is carried out "in situ", i.e. during the course of electrodeposition. Such an attachment for the "in situ" study of electrolytic coatings structure has been designed by Lagiewka and Budniok, 1986.

\section{SPECIMEN PREPARATION}

The models of our study were bright electrodeposited zinc layers. The deposition was carried out on different substrates (disk cathodes with a diameter of $32 \mathrm{~mm}$ ), namely: annealed (specimen No. 1) and nonannealed (specimen No. 2) $\alpha$-Fe with a nearly random distribution of crystallites; electrodeposited nickel coatings with $\langle 110\rangle+\langle 411\rangle$ (specimen No. 3), $\langle 100\rangle+\langle 221\rangle,\langle 210\rangle+\langle 542\rangle$ texture the thickness of which was about $60 \mu \mathrm{m}$; amorphous $\mathrm{Ni}-\mathrm{Fe}-\mathrm{P}$ coatings (on $\alpha$-Fe substrates) with a thickness of about $10 \mu \mathrm{m}$ (specimen No. 4). The layers were plated in an electrolyte with the following composition: $\mathrm{ZnSO}_{4} \cdot 7 \mathrm{H}_{2} \mathrm{O} 110 \mathrm{~g} / \mathrm{l}$, $\mathrm{H}_{3} \mathrm{BO}_{3}-30 \mathrm{~g} / \mathrm{l}, \mathrm{NH}_{4} \mathrm{Cl}-30 \mathrm{~g} / \mathrm{l}$. The brightener additives $\mathrm{ZC1}-35 \mathrm{ml} / 1$ and $\mathrm{ZC} 2-5 \mathrm{ml} / \mathrm{l}$ were employed as described in a previous investigation (Nikolova $e t$ al. 1990). The temperature of the bath was $20^{\circ} \mathrm{C}$ and $\mathrm{pH}=4.5$. The sublayers were deposited at cathodic current density $2 \mathrm{~A} \cdot \mathrm{dm}^{-2}$. The final thickness of each $\mathrm{Zn}$ specimen of about $60 \mu \mathrm{m}$ was obtained as a result of successively grown sublayers. The thickness of each sublayer was determined by means of the weight method. The time between two consecutive depositions was about 20 min. Moreover, it is assumed that the deposition interruptions have no effect on the layer texture evolution, i.e. the final texture is identical to the one formed if the deposition was carried out without interruptions. The use of such an approach for obtaining the specimen in the concrete case is possible because of the strong homoepitaxy of zinc (Tomov et al. 1989).

The measurements of the $\{10 \overline{1} 0\}$ and $\{11 \overline{2} 0\}$ diffraction lines of zinc were performed with an X-ray diffractometer Philips with $\mathrm{CuK}_{\infty}$ radiation ( $\mathrm{LiF}$ focusing monochromator).

\section{EXPERIMENTAL AND DISCUSSION}

In the most general case the texture arising in a layer may be divided into three stages: initial, transition and final texture (Bauer 1964). With respect to their appearance, there are two different standpoints considering the possible causes of texture arising in electrodeposited and vapour deposited layers: namely, oriented nucleation (Pangarov 1965) and oriented crystal growth (Gorbunova 1943, Laemmlein 1945, Bauer 1964, van der drift 1967). The role of each of them may 
TEXTURE EVOLUTION IN LAYERS

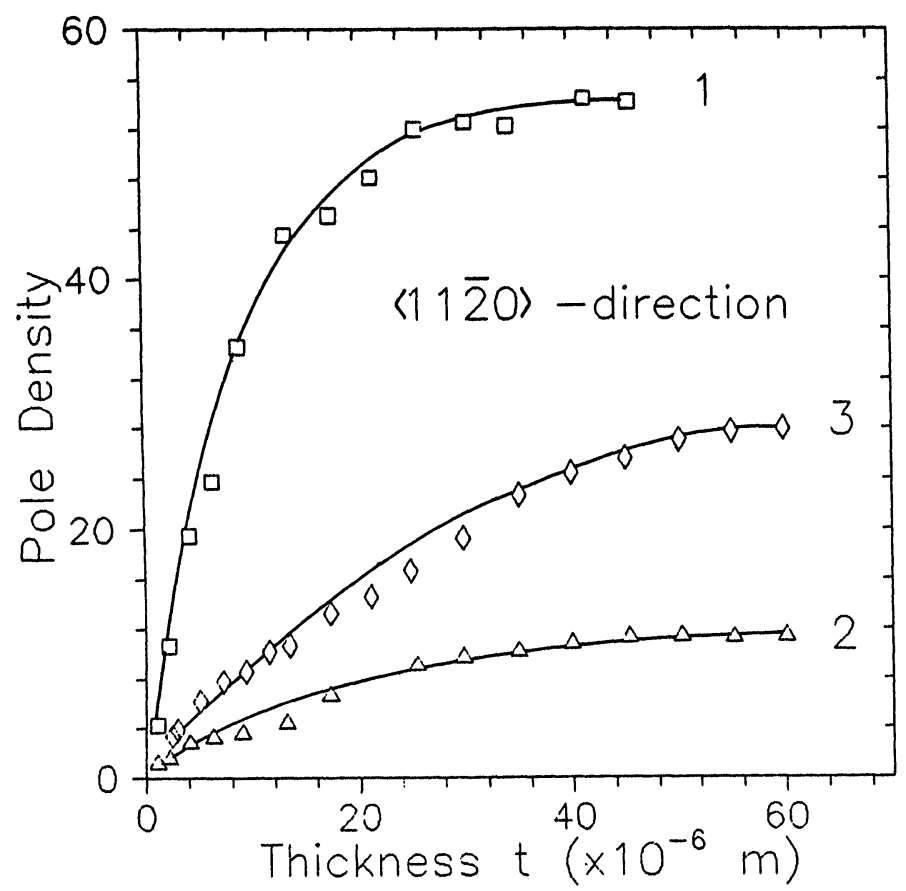

a.

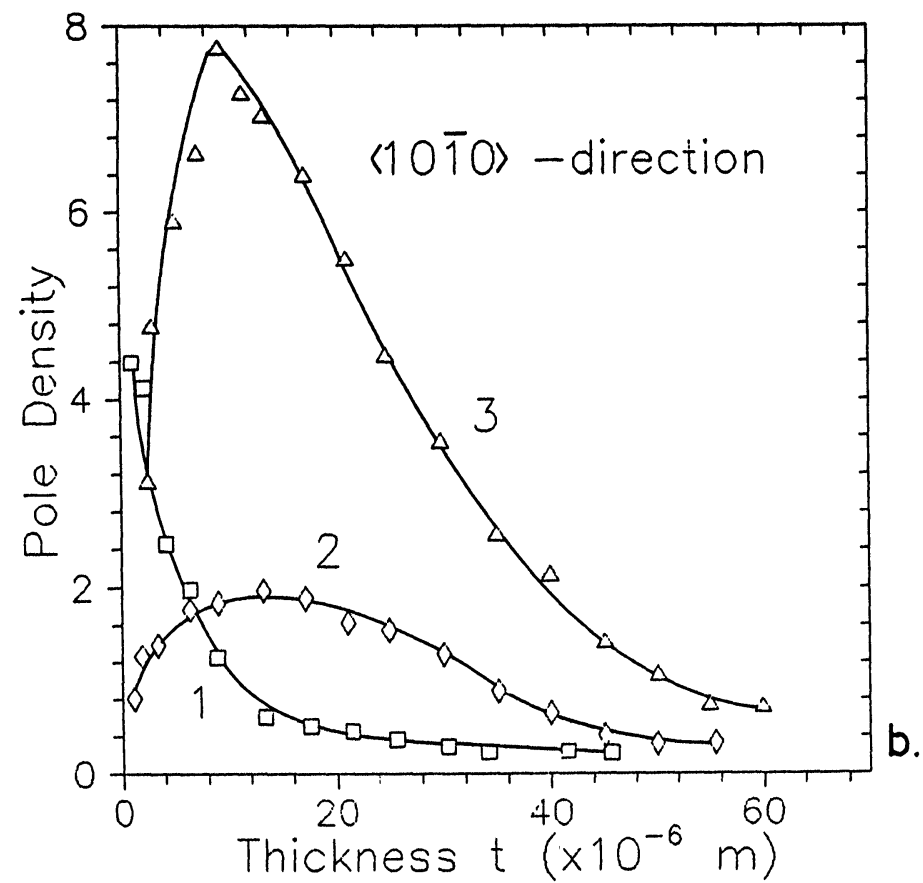

Figure 2 Dependence of pole density $P_{n}$ according to Eq. (7) vs. thickness $t$ for the $\langle 11 \overline{2} 0\rangle$ direction (a) and the $\langle 10 \overline{10}\rangle$ direction (b) for a specimen deposited on annealed iron substrate (curve 1), nonannealed iron substrate (curve 2), amorphous Ni-Fe-P substrate (curve 3). 
be checked with our method which enables the establishing of the pole density distribution in ideal directions of main texture components. Moreover, the pole density may be employed as a measure for the sharpness of the relevant texture component.

In the initial stage of the arising of texture (mainly at a thickness of about $1 \mu \mathrm{m}$-these are the first points of the curves given in Figures 2 and 3) were established only $\langle 10 \overline{1} 0\rangle$ and $\langle 10 \overline{2} 0\rangle$ preferential orientations both for the cases of orientated, non-oriented and amorphous substrates. This presumes that the type of initial orientations is determined by the electrochemical conditions. The ratio of the pole densities is in favour of the $\langle 11 \overline{2} 0\rangle$ component for the case of one of the oriented nickel substrates (Figure 3) and in favour of the $\langle 10 \overline{1} 0\rangle$ component-for all other cases. Thus it follows that the substrate has an effect on the ratio, but not on the type of orientations.

During the transition stage the $\langle 10 \overline{1} 0\rangle$ component disappears while the respective curves show a different course depending on the substrate structure. For annealed substrates (Figure $2 b$ curve 1 ) the $\langle 1010\rangle$ pole density sharply falls with the increase in coating thickness, while for amorphous and nonannealed substrates (Figures $2 \mathrm{~b}, 3$ ) its change passes through a maximum. The presence of maxima is possibly due to various deformations in the annealed and nonannealed substrates. During the transition stage the pole density of the $\langle 11 \overline{2} 0\rangle$ component

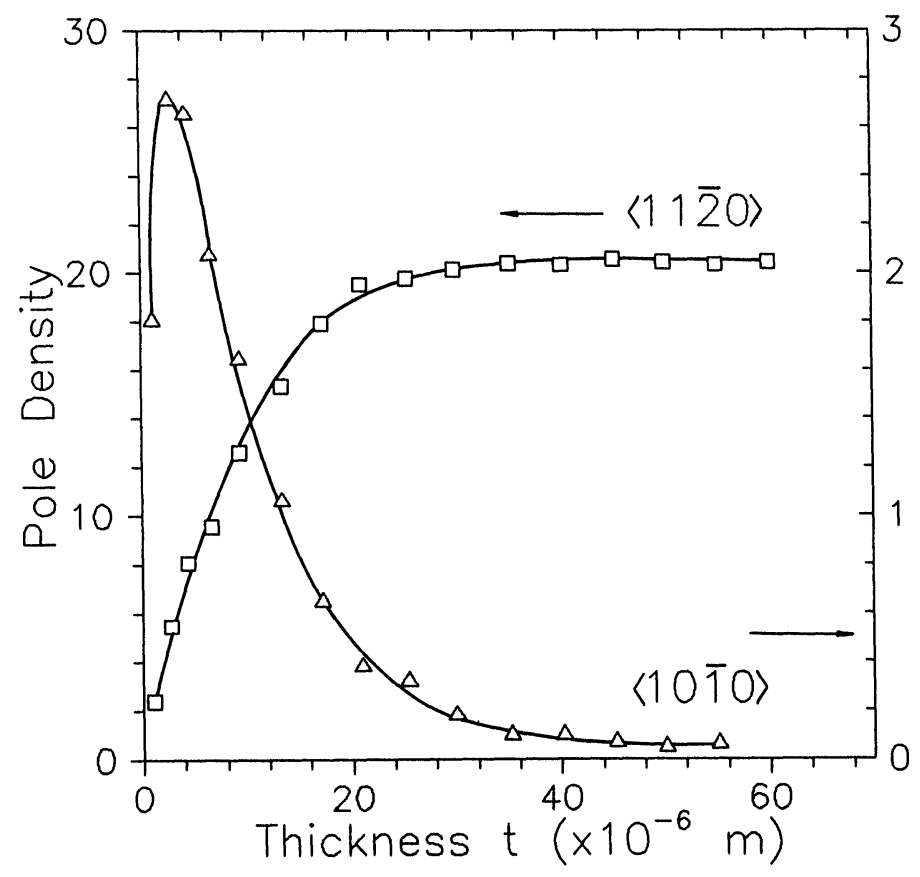

Figure 3 Dependence of pole density $P_{n}$ according to Eq. (7), vs. thickness $t$ for the $\langle 11 \overline{2} 0\rangle$ direction (compare the curve with the rectangles $\square$ to left ordinate axis) and $\langle 10 \overline{10}\rangle$ direction (compare the curve with the triangles $\Delta$ to right ordinate axis) deposited on nonannealed nickel substrated with a $\langle 110\rangle+\langle 411\rangle$ texture. 
grows continuously until it reaches a constant value, different for the different specimens.

The final texture has a single component $-\langle 11 \overline{2} 0\rangle$. Undoubtedly it is determined by the electrodeposition conditions.

The recapitulation of the experimental results suggests that during the texture formation in zinc coatings concurrent oriented nucleation occurs (two types of nuclei are mainly formed $\langle 10 \overline{1} 0\rangle$ and $\langle 11 \overline{2} 0\rangle$ on all kinds of substrates) and growth selection process (the final texture is a single component one). As a result of this process survive only the crystallites which are oriented in the direction of maximum growth rate normal to the substrate (Gorbunova 1943, Laemmlein 1945, van der Drift 1967). It thus follows that the final texture has been determined by the growth selection process, but it has originated from the initial nucleation. Hence the texture of the layer in all stages of its growth depends on the competition of the two processes. But the $\langle 10 \overline{10}\rangle$ pole density distribution of various specimens supposes that the substrate structure has an effect on the competition result (Figures $2 \mathrm{~b}, 3$ ).

No analytical equations are known which would express a dependence between the pole density (or the volume of crystallites with a given orientation) and the thickness of the layer. The observed course of the $\langle 11 \overline{2} 0\rangle$ curves have an experimental analogy (Pangarov et al. 1966). For the cases of electrodeposited Ni, $\mathrm{Co}, \mathrm{Cu}, \mathrm{Ag}$ layers Pangarov et al., 1966 established that the development of a definite orientation axis (main texture component) takes place to the thicknesses of about 2-3 $\mu \mathrm{m}$ (texture sharpness increase during the vertical growth). The authors have explained their results from the standpoint of oriented nucleation. This explanation contradicts in principle the summation of Bauer, 1956, and van der Drift, 1967, according to whom in the case of oriented nucleation the texture sharpness decreases during the vertical growth. What is more, according to our results, the texture development takes place to the thicknesses of about 20-30 $\mu \mathrm{m}$.

\section{CONCLUSION}

The method proposed by us allows to control the selective nucleation and growth of crystallites in chosen crystal directions of the layer. Thus the information acquired reveals the mechanism of texture formation. In fact, the results show definitely that there is a multicomponent texture in the initial and transition stages of texture formation. In all cases the final texture is a single component one, i.e. the texture arises during the growth of the layers. This reveals the role of growth selection process in texture formation.

The different course of the curves, showing the change of the $\langle 10 \overline{1} 0\rangle$ pole density, permits us to conclude that the texture generated depends on the substrate structure. Probably the various substrate structures influence the hydrogen evolution to a different degree and consequently, the nucleation process during the first stage of texture formation in the investigated models. 


\section{References}

Bauer, E. (1956). Z. Kristallogr. Kristallgeom., 107, 72.

Bauer, E. (1964). Single Crystal Films Symposium, Publication Division Pergamon Press, Oxford etc., pp. 43-67.

van der Drift, A. (1967). Philips Res. Repts, 22, 267.

Gorbunova, K. (1943). Tr. Vtoroi Konf. Corroz. Metal., Moskow, 2, 142.

Lagiewka, E. and Budniok, A. (1984). Surface and Coat. Technol., 27, 57.

Laemmlein, G. (1945). Dokl. Akad. Nauk. USSR, (Physics) 48, 177.

Nikolova, M., Raichevski, G., Banova, R. and Rashkov, S. (1989). B. Electroch., 5, 314.

Pangarov, N. A. (1965). J. Electroanal. Chem., 9, 70.

Pangarov, N. A., Vitkova, S. and Usunova, I. (1966) Electrochem. Acta, 11, 1747.

Reimer, L. and Freking, K. (1965). Z. Phys., 184, 119.

Tomov, I. Phys. stat. sol. (a), (1986) 95, 397; (1986) 98, 43.

Tomov, I. and Bunge, H. J. (1979). Texture Cryst. Sol., 3, 73.

Tomov, I. and Bunge, H. J. (1991)-to be published.

Tomov, I. , Riesenkampf, A., Pawlik, B., Cvetkova, Chr. and Velinov, V. (1989). J. Appl. Electrochem., 19, 377. 